\title{
The impact of community-wide, mass drug administration on aggregation of soil-transmitted helminth infection in human host populations
}

\author{
Marleen Werkman ${ }^{1,2,3^{*}}$ D, James E. Wright ${ }^{1,2,3,4}$, James E. Truscott ${ }^{1,2,3}$, William E. Oswald ${ }^{5}$, Katherine E. Halliday ${ }^{5}$,
} Marina Papaiakovou ${ }^{1,3,6}$, Sam H. Farrell1, Rachel L. Pullan ${ }^{5}$ and Roy M. Anderson 1,2,3

\begin{abstract}
Background: Soil-transmitted helminths (STH) are intestinal parasites estimated to infect over 1.5 billion people. Current treatment programmes are aimed at morbidity control through school-based deworming programmes (targeting school-aged children, SAC) and treating women of reproductive age (WRA), as these two groups are believed to record the highest morbidity. More recently, however, the potential for interrupting transmission by treating entire communities has been receiving greater emphasis and the feasibility of such programmes are now under investigation in randomised clinical trials through the Bill \& Melinda Gates Foundation funded DeWorm3 studies. Helminth parasites are known to be highly aggregated within human populations, with a small minority of individuals harbouring most worms. Empirical evidence from the TUMIKIA project in Kenya suggests that aggregation may increase significantly after anthelminthic treatment.

Methods: A stochastic, age-structured, individual-based simulation model of parasite transmission is employed to better understand the factors that might induce this pattern. A simple probabilistic model based on compounded negative binomial distributions caused by age-dependencies in both treatment coverage and exposure to infection is also employed to further this understanding.

Results: Both approaches confirm helminth aggregation is likely to increase post-mass drug administration as measured by a decrease in the value of the negative binomial aggregation parameter, $k$. Simple analytical models of distribution compounding describe the observed patterns well.

Conclusions: The helminth aggregation that was observed in the field was replicated with our stochastic individualbased model. Further work is required to generalise the probabilistic model to take account of the respective sensitivities of different diagnostics on the presence or absence of infection.
\end{abstract}

Keywords: Soil-transmitted helminths, Aggregation, Stochastic simulations

\footnotetext{
*Correspondence: m.werkman@imperial.ac.uk

${ }^{1}$ London Centre for Neglected Tropical Disease Research (LCNTDR),

Department of Infectious Disease Epidemiology, St. Mary's Campus,

Imperial College London, London, UK

Full list of author information is available at the end of the article
}

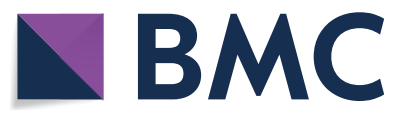

(c) The Author(s) 2020. This article is licensed under a Creative Commons Attribution 4.0 International License, which permits use, sharing, adaptation, distribution and reproduction in any medium or format, as long as you give appropriate credit to the original author(s) and the source, provide a link to the Creative Commons licence, and indicate if changes were made. The images or other third party material in this article are included in the article's Creative Commons licence, unless indicated otherwise in a credit line to the material. If material is not included in the article's Creative Commons licence and your intended use is not permitted by statutory regulation or exceeds the permitted use, you will need to obtain permission directly from the copyright holder. To view a copy of this licence, visit http://creativeco mmons.org/licenses/by/4.0/. The Creative Commons Public Domain Dedication waiver (http://creativecommons.org/publicdomain/ zero/1.0/) applies to the data made available in this article, unless otherwise stated in a credit line to the data. 


\section{Background}

The soil-transmitted helminths (STH) are a group of intestinal parasites comprising Ascaris lumbricoides (roundworm), Trichuris trichiura (whipworm), and Necator americanus and Ancylostoma duodenale (the hookworms). STH are thought to induce the largest morbidity burden of all neglected tropical diseases (NTDs) [1], with chronic medium- to high-intensity infections associated with growth retardation and intellectual impairment in children, and anaemia. There are an estimated 1.5 billion individuals infected with at least one intestinal nematode globally, cumulatively resulting in over five million disability-adjusted life years (DALYs) [2]. The greatest burden of STH infection falls upon those of lowest socioeconomic status in Southeast Asia and sub-Saharan Africa [3]. The most common anthelmintic drugs in use against STH are albendazole and mebendazole. Both are known to have few side effects $[4,5]$ generally high efficacy [6], and are currently donated for global use in deworming programmes by GlaxoSmithKline and Johnson \& Johnson, respectively.

The World Health Organisation (WHO) STH treatment guidelines currently target morbidity control, primarily through repeated, school-based de-worming programmes and aim to treat women of reproductive age (WRA) [7]. However, finding and treating WRA can be challenging [8]. More recently, however, a debate has started around the possibility of eliminating transmission, in addition to morbidity control, by expanding treatment across a broad class of age groups including, crucially, the adult population. This shift in focus was in part triggered by the London Declaration in 2012 which brought attention to the control of NTD morbidity by 2020 , and possible eradication of transmission of certain infections in some settings [9].

Previously published results have shown that interruption of transmission is unlikely to be achieved when only treating school-aged children (SAC) and WRA, since the untreated adult population will continue to contribute to the reservoir of infectious material thus causing reinfection amongst SAC [10-13]. This is especially the case for hookworm, where the majority of worms are harboured by adults [13], but it also applies to a lesser extent for the other prevalent STH species A. lumbricoides and T. trichiura [10]. It is important to note that even though WRA suffer more from morbidity than the adult male population, untreated adult males may harbour significant worm populations. Therefore, just treating SAC and WRA is unlikely to result in the interruption of transmission.

Successfully interrupting transmission would stop the need for long-term MDA in areas of endemic infection. Faced with the prospect of a never-ending dependence on
MDA, countries are starting to consider expanding deworming efforts beyond current school-based approaches [14-17]. As a result, greater emphasis has been placed upon implementing community-wide mass drug administration (MDA) de-worming programmes. The impact of successive rounds of MDA on STH prevalence is the primary focus of the Bill \& Melinda Gates Foundation funded DeWorm3 project in India, Malawi and Benin [18]. This project is investigating whether a communitywide MDA-only approach to STH treatment, would be sufficient to reach a prevalence threshold below which helminth parasite populations cannot sustain transmission, thus leading to transmission elimination [19-21]. All STH species reproduce sexually within a human host to produce fertile eggs. When the mean worm burden in a population falls to very low levels, the likelihood of both a male and female worm residing within a single human host becomes very small. A "breakpoint" is reached when the worm population can no longer sustain reproduction, resulting in the interruption of transmission even in the absence of further treatment $[20,21]$.

Helminth parasites are highly aggregated within human populations, with a small minority of individuals harbouring the majority of worms [22-27]. This "clumping" increases the likelihood that parasites successfully locate members of the opposite sex within a human host. Hence, the breakpoint in infection is much lower when parasites are aggregated as compared to randomly distributed throughout the host population. The aggregation parameter of the negative binomial distribution, $k$, inversely measures the extent of "clumping" or worm aggregation present in the human host population. The smaller the value of $k$, the greater the extent of aggregation [13]. As MDA coverage intensifies in endemic regions, it becomes more important to understand how the increased treatment pressure impacts the pattern of parasite aggregation, and whether any changes can help provide information to assist in improving the design of community-based MDA programmes. In this study, we investigate the impact of MDA on worm aggregation via observed patterns in communities experiencing high MDA coverage; through the predictions of probability models of the impact of MDA; and through individualbased stochastic simulations of parasite transmission and MDA impact under different patterns of individual compliance to multiple rounds of treatment.

Previous work has suggested that the probability with which repeated rounds of MDA will successfully result in transmission elimination is highly dependent on both treatment coverage, i.e. the proportion of individuals taking medication at any one round of treatment [28], and treatment compliance, i.e. the proportion of MDA rounds that each individual takes treatment $[29,30]$. Past 
research has shown that the impact of a communitybased MDA programme is greatly influenced by the specific pattern of non-compliance [30]. At one end of the spectrum it could be random, where all individuals have an equal chance of being treated at each round. At the other extreme is systematic non-compliance whereby the same individuals do not take treatment at any round of MDA. An intermediary pattern is semi-systematic in which each individual is assigned a lifelong probability of taking treatment at each round [30, 31]. Each of these non-compliance patterns may affect observed parasite aggregation patterns. Therefore, the analyses reported in this paper also examine the impact of different MDA compliance scenarios on the aggregation of parasites within the human host.

The overall aim of this study is to determine whether repeated, community-wide anthelminthic treatment has an impact on the degree of parasite aggregation and, if so, whether this impact can be predicted as a function of MDA coverage and compliance. We also discuss the policy implications of increased parasite aggregation post-MDA, as the prevalence of infection declines to low levels.

\section{Methods}

Empirical data derived from a large-scale field epidemiological study provided the stimulus for this research on parasite aggregation. The TUMIKIA project was a cluster-randomised, controlled trial conducted in Kwale County, Kenya, from March 2015 to May 2017. The Kwale County has received MDA to control lymphatic filariasis, which is also effective for the control of STH. Annual school-based MDA has been provided to this county three years before the start of the trial. The trial compared the impact on STH infection of annual and biannual MDA, versus annual mass anthelmintic treatment of children (2-14 years-old) [32, 33]. Recently, Truscott et al. [34] investigated the heterogeneity in transmission parameters from the TUMIKIA trial. Data obtained from the TUMIKIA study provided evidence of changes in the negative binomial aggregation parameter $k$, under intensive MDA. Clusters with a low prevalence were found to have a higher degree of parasite aggregation [34] (Fig. 1). In this study, we further investigate the changes in parasite aggregation. Since observations from the TUMIKIA study related to $N$. americanus, this will be the parasite of reference in this paper. However, results relating to $A$. lumbricoides will also be summarised, with further detail and figures provided in Additional file 1: Figures S1-S5.

We employ an event-driven, individual-based stochastic model of helminth transmission [30], building on a model framework originally described by Anderson \& May [35] and Anderson \& Medley [36]. The model

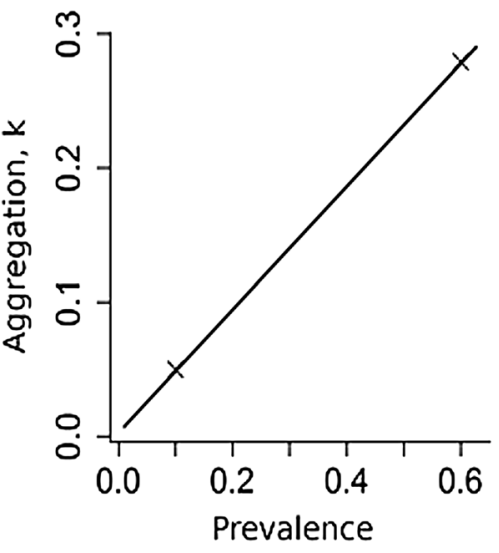

Fig. 1 Observed relationship between measured prevalence and aggregation, $k$, from TUMIKIA project. Originally published in Truscott et al. [34]. The crosses in represent the prevalence values of 10\% and $60 \%$. In the TUMIKIA study, the lowest $k$-values were found in low prevalence clusters $(<10 \%)$ and the highest $k$-values were found in clusters with a prevalence of approximately $60 \%$

includes age-dependent infection rates, density-dependent worm fecundity, parasite sexual reproduction which is assumed to be polygamous, and a negative binomial distribution of parasites per person [37]. The latter is created by assigning an exposure risk factor to individuals drawn from a gamma distribution for a specific age-grouping (the compounding of Poisson distributions where the means are drawn from a gamma distribution [38]). It is the dynamics of sexual reproduction for dioecious species which results in the occurrence of three possible equilibria in the deterministic dynamical system; a stable endemic state, and parasite extinction, separated by an unstable "breakpoint" in transmission below which the attractor is extinction [27]. We denote this as a prevalence threshold below which the parasite population cannot sustain transmission. Once this state is reached, there is no need for further MDA assuming an absence of infective individuals migrating to the area who could re-populate the reservoir of infection. In a stochastic world, the deterministic concept of a breakpoint needs expansion to a small area around the deterministic breakpoint where the system, due to chance events, may move to either stable equilibrium. The individual-based, stochastic modelling of events via numerical studies allows for the explicit inclusion of heterogeneity between individuals, such as in treatment compliance and exposure to infection, as well as probabilistic forecasts of the likely impact of different MDA programmes in a variety of transmission settings (e.g. low, medium and high transmission settings as defined by the value of the basic reproductive number, $\mathrm{R}_{0}$ ). 
The parameter values employed in the individual-based, stochastic simulation model for N. americanus and A. lumbricoides are presented in Table 1. Each stochastic modelbased simulation comprised 1000 villages, each containing 1000 individuals. All villages underwent five rounds of annual, community-wide MDA, with coverage levels for $N$. americanus set at $85 \%$ for both pre-school-aged children (pre-SAC) and SAC, and 75\% for adults, whilst for A. lumbricoides coverage was $75 \%$ for pre-SAC and SAC, and 30\% for adults. These coverage levels were selected to ensure that a proportion of simulations achieved elimination and a proportion did not, such that the validity of the predictions of the simple analytical model could be compared with those of the more complex individual stochastic model in both scenarios. Simulations were run for 10 years pre-treatment and 30 years post-treatment to ensure STH prevalence had reached equilibrium both before and after anthelmintic treatment was provided.

At each time point, the effective negative binomial aggregation parameter is calculated by finding the maximum likelihood estimate $\hat{k}$ from the distribution of worm burdens amongst hosts [39]. These estimates are subsequently presented as line graphs showing how worm aggregation changes over time. STH prevalence is also calculated for each village at each time points.

The impact of treatment on parasite aggregation can also be expressed in a simplified probability model from which some key analytical results can be derived. In a homogenous population, worm burdens among hosts are precisely negative binomial, with a fixed $k$ value within our simulation. However, once heterogeneities are introduced, such as age structure and differing uptake of MDA within the population, this is no longer the case. Worm burden distributions after MDA or across wide age ranges with differing exposure intensities can be described by a compound distribution, in which the underlying negative binomial distribution is compounded by the probability distribution associated with the given source of heterogeneity. Using this approach, we can approximate the impact of these mechanisms on aggregation by calculating the changes to both the mean and variance of the worm burden among hosts that they influence. For a negative binomial distribution, the relationship between the aggregation parameter $k$, the mean $\mu$ and the variance $\sigma^{2}$ is given by

$$
k=\frac{\mu^{2}}{\sigma^{2}-\mu} \text {. }
$$

Under the effect of heterogeneity, the new values of mean worm burden and variance can be used to calculate an effective aggregation parameter, $k^{\prime}$, under the assumption that the worm distribution is still approximately negative binomial.

In the case of MDA with imperfect coverage, a round of treatment splits the target population into two sub-populations: a treated group with reduced worm burden and an untreated group whose worm burden is assumed to be unchanged. Using the technique described by Anderson \& May [27], for a round of MDA, with coverage $c$ and efficacy $e_{f}$, applied to a homogeneous host sub-population with mean worm burden $m$ and aggregation $k$, the approximate aggregation post-treatment is

$$
k^{\prime} \simeq k \frac{\left(1-e_{f} c\right)^{2}}{(k+1) e_{f}^{2} c(1-c)+\left(1-e_{f} c\right)^{2}}
$$

By extending this approach, we can also incorporate the heterogeneity induced by age-dependent exposure to infection. For further information, please see Additional file 2: Text S1.

If we consider the impact of treatment across a range of ages where there is a significant difference in exposure to infection, a further level of variability is introduced

\begin{tabular}{|c|c|c|}
\hline Model parameter description & A. lumbricoides & N. americanus \\
\hline Transmission rate, $\mathrm{R}_{0}$ & 2.12 & 2.2 \\
\hline Aggregation of worms within human hosts, $k$ & 0.15 & $0.35[25]$ \\
\hline \multirow{4}{*}{$\begin{array}{l}\text { Relative exposure to infection and contribution to infective reservoir (assuming no differ- } \\
\text { ences between males and females) }\end{array}$} & $0-2$ years-old: 0.22 & $0-2$ years-old: 0.03 \\
\hline & 2-5 years-old: 1.88 & 2-5 years-old: 0.09 \\
\hline & 5-15 years-old: 1 & $5-15$ years-old: 1 \\
\hline & $15+$ years-old: 0.53 & $15+$ years-old: $2.5[49]$ \\
\hline Average worm life expectancy (years) (assuming an exponential distribution) & $1[50]$ & $2[51]$ \\
\hline Female worm fecundity, $y$ & $0.07[37]$ & $\begin{array}{l}0.02 \text { (assuming expo- } \\
\text { nential saturation) } \\
\text { [52] }\end{array}$ \\
\hline Infectious reservoir decay rate (mean) & 2 months [35] & 12 days [51] \\
\hline Drug efficacy & $0.99[40]$ & $0.948[40]$ \\
\hline
\end{tabular}

Table 1 Parameters used for the individual-based, stochastic simulation models 
into the calculation. If we construct $N$ age categories, the probability of sampling (randomly) from the $i^{\text {th }}$ is

$$
p(i)=\frac{\# \text { people in } i^{\text {th }} \text { age group }}{\text { Total population }}
$$

where the value of $\mathrm{m}_{0}$ and $c$ are functions of the agegroup $i$. The new variance of worm burden now becomes fully-systematic and semi-systematic non-compliance scenarios (range: $0.100-0.160$ and $0.100-0.151$, respectively). In all scenarios, $k$ became successively smaller after each round of treatment, with the recovery in $k$ immediately post-treatment consistently lower than at each previous round. Furthermore, the range in $k$ values recorded in the stochastic simulations increased slightly

$$
\left.\operatorname{Var}[w]^{\prime}=E_{i} E_{m}\left[\operatorname{Var}_{w}[w(m)]\right]\right]+E_{i}\left[\operatorname{Var}_{m}\left[E_{w}[w(m)]\right]\right]+\operatorname{Var}_{i}\left[E_{m}\left[E_{w}[w(m)]\right]\right]
$$

This can be expressed in terms of the previous simpler expression for the variance

$$
\begin{aligned}
\operatorname{Var}[w]^{\prime}= & E_{i}\left[\operatorname{Var}[w]\left(m_{0}(i), c(i)\right)\right] \\
& +\operatorname{Var}_{i}\left[\bar{m}\left(m_{0}(i), c(i)\right)\right]
\end{aligned}
$$

Since coverage levels and mean worm burden are both functions of age group, this expression cannot be evaluated in closed form.

The expected $k$ values, mean worm burdens and variance in worm counts immediately post-treatment are calculated from the pre-treatment data obtained from the simulations. These expected post-treatment values are then compared to those observed from the simulations to determine the validity of the simple analytical predictions. Scatterplots are used to visually portray the comparisons.

\section{Results}

\section{Predictions based on the stochastic simulations}

At equilibrium, $N$. americanus prevalence was approximately $65 \%$ in all villages for all compliance scenarios. After cessation of treatment, village prevalence either recovered to pre-treatment equilibrium levels or decreased to zero, depending on whether the breakpoint in transmission was crossed. The proportion of villages achieving $N$. americanus elimination was $89.4 \%$ under random non-compliance, $41.7 \%$ for semi-systematic compliance and $0 \%$ for fully-systematic compliance (Fig. 2a-c). For A. lumbricoides the equivalent proportions were $75.4 \%, 65.6 \%$ and $42.8 \%$, respectively (Additional file 1: Figure $\mathrm{S} 1 \mathrm{a}-\mathrm{c}$ ). These results clearly illustrate the importance of individual compliance as a key determinant of MDA impact. This effect is not widely appreciated by those who track MDA coverage as reported on a country to country basis to WHO. In the WHO compilations of year by year progress in MDA, coverage is documented but not compliance.

Under random non-compliance, $k$ decreased sharply (reflecting increased parasite aggregation) to between 0.102 and 0.160 directly following the first round of treatment. Similar patterns were also observed in both the after each successive round of treatment. In villages where elimination of transmission was successful (the "breakpoint" in transmission was crossed), the prevalence declined to zero and $k$ concomitantly decayed to a value of zero. On average, this took 12 years post-cessation of treatment for random non-compliance (range: $3.0-21.0$ years) and 14.5 years for semi-systematic (range: 6.5-21.0 years). No simulations in the fully-systematic non-compliance scenario resulted in elimination, hence no $k$ values reached zero.

For A. lumbricoides, the duration of time for $k$ to reach zero post-treatment was 6.5 years for random non-compliance (range: 0.5-20.0 years), 7.0 years for semi-systematic compliance (range: 1.5-19.5 years), and 8 years for fully-systematic non-compliance (range: $3-21.0$ years). In those villages which failed to reach elimination and suffered bounce-back in infection to pre-treatment equilibrium levels, $k$ was observed to also return to its pre-treatment value. On average, this bounce-back took approximately 6 years post-cessation of treatment for both $N$. americanus (Fig. $2 \mathrm{~d}-\mathrm{f}$ ) and A. lumbricoides (Additional file 1: Figure S1d-f). No significant difference was observed between different compliance patterns with regards to the duration of time required for $k$ to return to pre-treatment equilibrium levels.

The short- and long-term impact of treatment can also be observed in the age-prevalence surface plots (the dimensions of age and time). At equilibrium, the prevalence of hookworm infection was higher amongst adults than pre-SAC and SAC. Treatment resulted in a decrease in prevalence amongst all age groups, with the greatest relative decrease observed in adults. In villages which did not reach elimination and bounced-back to the pretreatment equilibrium, the post-treatment age-prevalence profiles were near-identical to pre-treatment levels (Fig. 3, Additional file 1: Figure S2).

The proportion of individuals who still harbour worms after MDA showed differences between the compliance patterns types (Additional file 1: Figure S3). At round five, the proportion of individuals who harbour at least one worm were $5.1 \%(0.8-12.5 \%), 7.8 \%(2.9-16.6 \%)$ and 

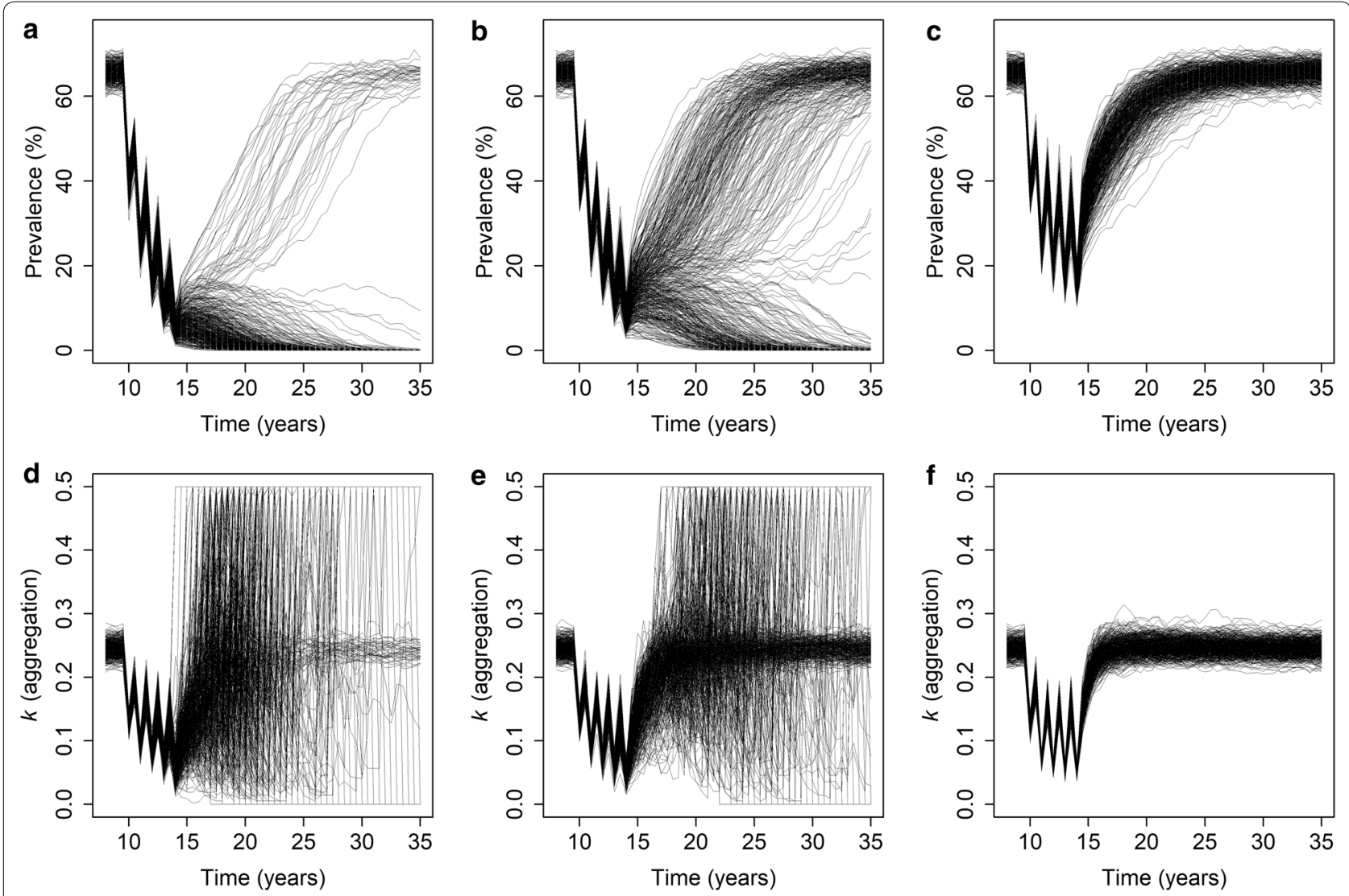

Fig. 2 Prevalence of Necantor americanus over time (a-c) and aggregation (parameter $k$ ) over time (d-f), in this plot we show 500 out of 1000 simulated villages. $\mathbf{a}$ and $\mathbf{d}$ : Random non-compliance; $\mathbf{b}$ and $\mathbf{e}$ : Semi-systematic non-compliance; c and f: Fully-systematic non-compliance

$16.1 \%(10.4-24.1 \%)$ for random, semi-systematic and fully-systematic non-compliance (Additional file 1: Figure S3). The proportion of individuals who harbour both male and female worms reduced on average to $1.2 \%$ (0-4.5\%) with random compliance (Fig. 4). With fullysystematic non-compliance, the proportion of individuals with both male and female worms remained high even after five rounds of MDA (8.4\%, 5.0-13.4\%) allowing transmission to be sustained. Similar results were found for A. lumbricoides (Additional file 1: Figures S3, S4).

\section{Simple theory compared with the outcomes predicted by the complex stochastic simulations}

The impact of MDA on the degree of aggregation was found to be described well by the simple probability model of compounding distributions created by heterogeneities within the host population due to either compliance to treatment or exposure to infection. For $N$. americanus, a high degree of agreement was seen between the mean worm burden and $k$ values observed from the stochastic simulations immediately post-treatment and those expected under the simple probability model defined by the equations given in Additional file 2: Text S1. Strong agreement between observed and expected results was identified for A. lumbricoides, with the variance in worm count post-treatment showing a much stronger agreement between models than was found for N. americanus (Fig. 5; Additional file 1: Figure S5).

\section{Discussion}

We have shown that the aggregation parameter $k$ decreases immediately after each round of treatment reflecting increased parasite aggregation within the human host population. Furthermore, this decrease can be accurately predicted using a simple probability model which incorporates heterogeneities within human host populations created by either age-dependencies in worm burden (i.e. age-dependent exposure to infection) and/ or variation in treatment coverage by age. In the longterm over multiple rounds of MDA, the decline in the value of $k$ follows the decline in prevalence within any given village. Our results show that the A. lumbricoides simulations are more likely to achieve interruption of 

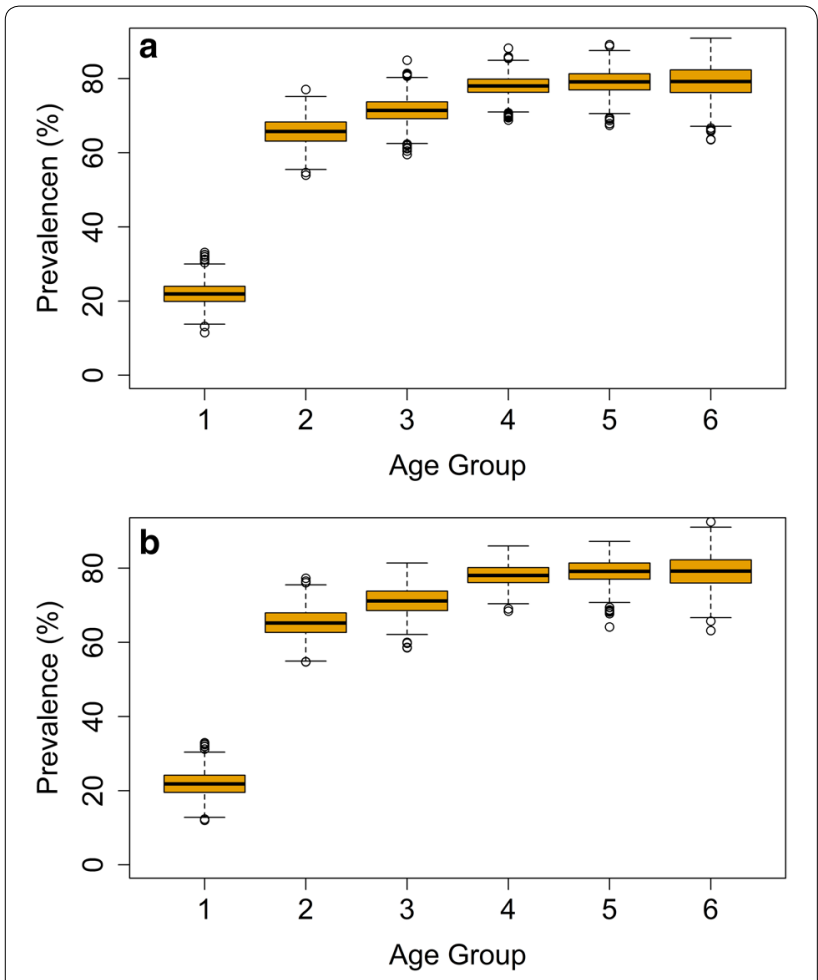

Fig. 3 Age-prevalence over time in which elimination of Necator americanus infection was not achieved before mass drug administration was implemented (a) and post-MDA (b). Age groups (years): $1,0-5 ; 2,6-10 ; 3,11-15 ; 4,16-30 ; 5,31-50 ; 6,51+$

transmission for all three compliance types. This is likely to be the result of higher coverage in the SAC-population which have typically the highest $A$. lumbricoides worm burden. Whilst for hookworm the opposite is noticed.
Moreover, the egg reduction rate is slightly higher for $A$. lumbricoides [40].

The simple model requires data describing the number of worms within each individual host both pre- and post-treatment. Worm expulsion post treatment is both time- and resource-intensive, by comparison with collecting data on egg counts via stool examination (typically through Kato-Katz, KK). Worm expulsion data can be unreliable since stool samples need to be collected for at least 6 consecutive days to collect at least $80 \%$ of A. lumbricoides worms post-treatment [41]. Given that hookworms are much smaller than A. lumbricoides, worm counts are considered even less precise as many worms are likely to be missed in the collected stool. Since worm expulsions are performed by giving individuals chemotherapeutic treatment, the collection of empirical "pre-treatment" worm counts is often not possible in current government run deworming treatment programmes. We can extract information on worm burden at any time point from the individual-based stochastic simulations models, but such data are difficult to obtain in ongoing field epidemiological studies. One potential solution (although by no means a perfect one) could be to compare post-treatment worm expulsion data with pre-treatment KK intensity data for the same individuals. Whilst this would not be "worm expulsions" at both time points and the two measures would not necessarily be directly comparable, this approach may be of help given good data on the relationship between worm burdens and egg counts.

Egg count data, collected at both pre- and post-treatment, can be used as a proxy for worm count data. However, microscopic diagnostic tools, such as KK, have a reduced sensitivity at low intensities of infections.
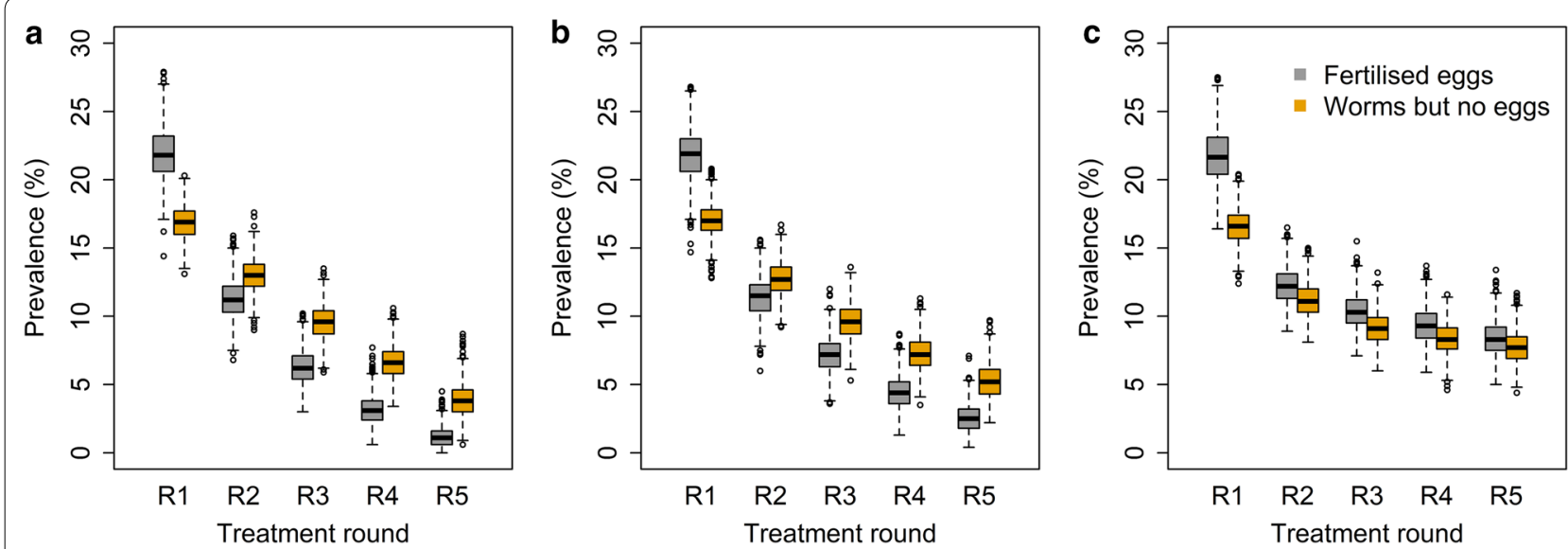

Fig. 4 The proportion of individuals who harbour both male and female worms and produce fertile eggs (grey shaded color) and the proportion of individuals who harbour either only male or only females worms and produce no eggs (orange shaded color) for random compliance (a), semi-systematic compliance (b) and systematic compliance (c) 

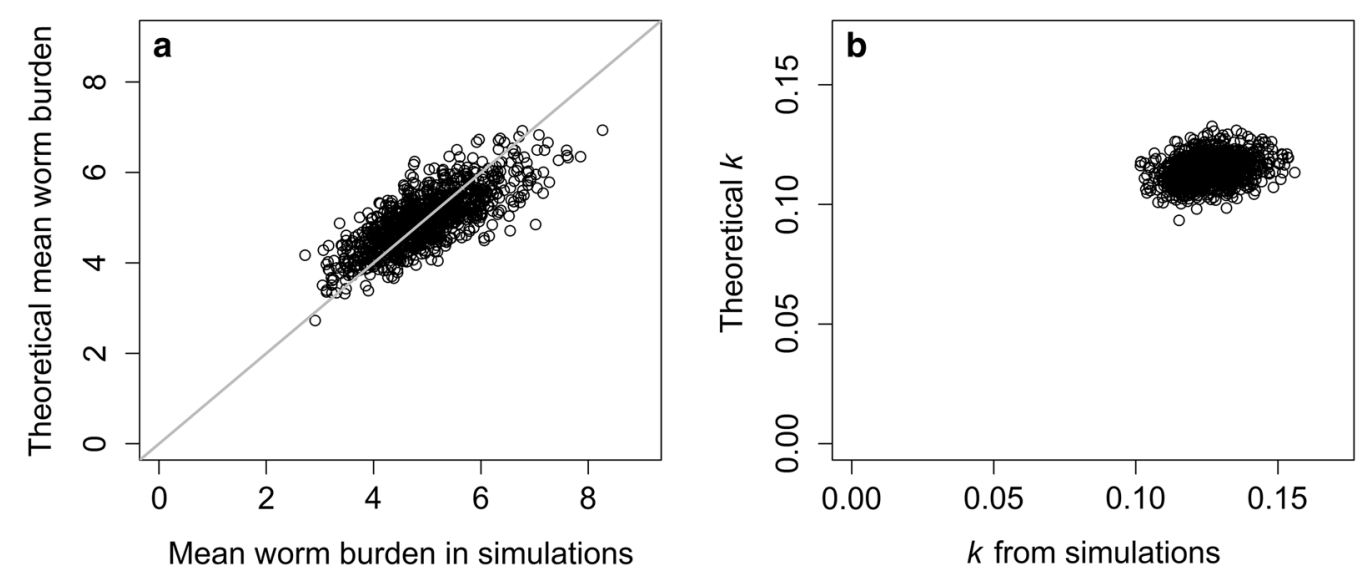

Fig. 5 Results from analytical approach for predicting change in aggregation after treatment under semi-systematic non-compliance. a Comparison of mean worm burden observed from the stochastic model immediately post-treatment and that expected from the simple probability model. $\mathbf{b}$ Comparison of $k$ observed from the stochastic model immediately post-treatment and that expected from the simple probability model. Note: for $\mathbf{a}$, the grey line represents the equation $\mathrm{x}=\mathrm{y}$ (perfect agreement)

Nikolay et al. [42] showed that whilst the sensitivity of double-slide KK (the most commonly used microscopic diagnostic tool) was acceptable for the three major STH species in high-intensity of infection areas (sensitivity of 74-95\%), it was much less reliable in areas of low intensity (sensitivity of $53-80 \%$ ), with the lowest values being for hookworm and A. lumbricoides. Therefore, these diagnostic tools are less suitable for populations that are close to reaching the breakpoint in transmission. The increasing use of MDA combined with the ongoing push for STH elimination will likely result in greater pressure on the use of more sensitive diagnostics to detect infection at lower prevalence and intensity. To this end, there has been increased interest in recent years in the potential use of quantitative polymerase chain reaction (qPCR) assays as a diagnostic tool for STH infections [43, 44]. This technique has greater sensitivity than KK especially when the prevalence and intensity of infection are both low $[20,43,44]$. The use of improved diagnostics with increased sensitivity will be very beneficial in areas approaching the breakpoint in transmission to ascertain if transmission has ceased. However, the increased costs of qPCR by comparison with KK may be a deterrent in low resource settings.

Calculating $k$ values was found to be difficult for some villages in both the random and semi-systematic noncompliance simulations post-cessation of treatment due to very low average worm burdens such that very few individuals were infected. When all individuals within a village have either zero or one worm, as was the case in the villages for which $k$ could not be estimated, the negative binomial distribution no longer describes the worm burden distribution and a Bernoulli distribution is a more appropriate probability model. In these circumstances, no negative binomial aggregation parameter could be derived and the maximum likelihood analysis was forced to select whichever value was set as the upper limit for $k$ as the best fit (here, $k=0.5$ ) in the estimation process. A fuller explanation is presented in Additional file 2: Text S1.

Aggregation of macroparasites within the human hosts is a commonly observed epidemiological feature. The degree of overdispersion can depend on the parasite species, host age and gender, and the transmission setting. For example, children are often predisposed to heavy $A$. lumbricoides infection. Predisposition to hookworm infection is also observed, but the factors that are linked to predisposition are not well understood at present [45]. It may be some combination of genetic, behavioural and environmental factors.

Understanding compliance within a study through the collection of individual-based, longitudinal treatment data is of the upmost importance. The probability of successfully eliminating $N$. americanus infection within our simulations with a fixed level of coverage was highly dependent on the pattern of compliance assumed, ranging from almost $90 \%$ with random compliance to $0 \%$ under systematic non-compliance. Therefore, it is crucial to the success of a deworming campaign aimed at breaking transmission that all individuals within a village are treated and that no individuals are systematically missed. This can occur when households are too remote, individuals persistently refuse treatment or if individuals are consistently not at home when health care workers are visiting. Knowledge of the factors that influence persistent non-compliance 
can help in the design and implementation of parasite control programmes.

Knowledge regarding the extent of compliance within a population targeted for treatment can contribute towards an understanding of whether or not to stop randomised STH control trials, such as DeWorm3, earlier than originally planned $[18,21]$. If compliance is consistently poor there is little point in continuing treatment, monitoring and evaluation since transmission elimination is unlikely to occur.

Anthelmintic resistance is a significant problem in livestock farming and is increasingly reported amongst by the veterinary profession. It is important to note that drug pressure in livestock is much higher, in part as a consequence of livestock being treated five times a year and hence parasites have a high and repeated exposure to the drug selective pressure. Nonetheless, the risk of developing drug resistance in human STH is important to monitor when the goal is shifting from morbidity control towards interruption of transmission. This is especially true for species with a suboptimal response to the drugs, for example the response of T. trichiura to albendazole and mebendazole. With the shift towards interruption of transmission, drug pressure within a population increases substantially and concomitantly selection pressure on the parasite population. Even though there is widespread agreement regarding the importance of monitoring drug resistance, reliable studies investigating this challenge are very few at present. Microscopic diagnostic tools and qPCR alone are not sufficient to identify drug resistance, other methods such as population genetics are required. Worm DNA needs to be isolated from expulsion studies and the beta-tubulin gene (where resistance has been reported before) needs to be sequenced. One example of recent progress is the STARWORM project which aims to identify standardized methods to monitor drug resistance [46]. During the DeWorm3 trial drug resistance is being closely monitored and samples are being stored to investigate resistance mutations in future molecular epidemiological analyses. If drug resistance appears to develop in the DeWorm3 study sites, it will be important to understand why this is the case and monitoring changes in aggregation patterns can support the detection of drug resistance [18].

The effects of migration of infectious individuals on the likelihood of interrupting transmission are poorly understood at present for helminth control. However, much recent infectious disease research is focused on this issue employing simple or complex models of spatial structure and human and animal movement. In this study, we assumed that villages behave as independent units with no immigration or emigration of infectious individuals. Recent molecular epidemiological analyses performed by Easton et al. [47], showed that for $A$. lumbricoides infection in Bungoma (Kenya), the village is the most appropriate epidemiological unit for study, since molecular epidemiological analyses revealed that the vast majority of transmission takes place between village members. If there is a lot of migration between villages, the risk of transmission from one village to another increases. However, this risk will depend on a number of factors, including the prevalence of infection and its heterogeneity between villages, heterogeneity in treatment coverage and compliance between villages, the age-group that moves most between villages and also the age-intensity profiles. For example, if there is a high prevalence of $A$. lumbricoides in one village, there is a low risk of transmission between villages as a consequence of migration if adults are the major migratory group. Whilst for hookworm the opposite is true and reaching a breakpoint will be difficult if much adult movement takes place [48]. Coverage and compliance on the village-level or household-level are obviously of high importance. If for example, a few households refuse to take the treatments offered in MDA rounds, they may maintain a local reservoir of infection within a village, which can complicate reaching the transmission breakpoint. How serious this complication is, depends on the contact of other village members with non-compliant households. Data currently being collected during the DeWorm3 trials will be very beneficial in parameterizing spatially structured models and in refining programmes that aim to interrupt transmission of STH on large spatial scales.

In future research, it is important to adapt the methods outlined in this paper to allow for the use of egg counts (eggs per gram of faeces; EPG) as opposed to the worm counts (which require high resource allocation to ensure accuracy). Worm expulsion studies are considerably more time- and resource-intensive than obtaining faecal egg counts via Kato-Katz. Adapting the simple model, such that EPG data can be utilised, will allow for the methodology to be used more widely within STH studies.

\section{Conclusions}

We have shown how the increased aggregation in STH infection post-treatment observed in empirical studies can be predicted using a simple probability model based on compounding distributions arising from heterogeneities due to age-dependent exposure to infection and/or different patterns of MDA uptake by individual people. Ongoing studies under the TUMIKIA and D3Worm3 projects will provide further information 
on patterns of individual compliance. For hookworm, infection individual compliance is thought to be the major cause of the observed patterns of increasing parasite aggregation with increasing numbers of MDA rounds in given communities. Increased aggregation of worms after many rounds of chemotherapy suggests that once the prevalence of infection has reached low levels an approach to target and treat those predisposed to infection and non-compliant to treatment may be highly beneficial.

\section{Supplementary information}

Supplementary information accompanies this paper at https://doi. org/10.1186/s13071-020-04149-4.

Additional file 1: Figure S1. A. lumbricoides prevalence over time (a-c) and aggregation (parameter $k$ ) over time $(d-f)$, in this plot we show 500 out of 1000 simulated villages. $a$ and $d$ : Random non-compliance; $b$ and e: Semi-systematic non-compliance; $c$ and f: Fully systematic noncompliance. Figure S2. Age-prevalence over time for a selected village in which elimination of A. lumbricoides infection was not achieved before mass drug administration was implemented (a) and post-MDA and one in which elimination was not achieved (b). Age groups (years): 1, 0-5; $2,6-10 ; 3,11-15 ; 4,16-30 ; 5,31-50 ; 6,51+$. Figure S3. The proportion of individuals who still harbour worms, either hookworm (a) or $A$. lumbricoides (b) after each round of mass drug administration (T1-5) for random compliance, semi-systematic compliance and systematic compliance. Figure S4. The proportion of individuals who harbour both male and female worms (A. lumbricoides) and produce fertile eggs (grey shaded color) and the proportion of individuals who harbour either only male or only females worms and produce no fertile eggs (orange shaded color) for random compliance (a), semi-systematic compliance (b) and systematic compliance (c). Figure $\mathbf{S 5}$. Results from analytical approach for predicting change in aggregation after treatment under semi-systematic non-compliance. a Comparison of mean worm burden observed from the stochastic model immediately post-treatment and that expected from the simple probability model. b Comparison of $k$ observed from the stochastic model and that expected from the simple probability model immediately post-treatment. Note: For $\mathrm{a}$, the gray line represents the equation $\mathrm{x}=\mathrm{y}$ (perfect agreement).

Additional file 2: Text S1. A simple analysis of the impact of treatment and heterogeneity on worm aggregation among hosts.

\section{Abbreviations}

DALYs: disability-adjusted life years; EPG: eggs per gram of faeces; KK: KatoKatz; MDA: mass drug administration; NTDs: neglected tropical diseases; SAC: school-aged children; STH: soil-transmitted helminths; qPCR: quantitative polymerase chain reaction; WHO: World Health Organisation; WRA: women of reproductive age.

\section{Acknowledgements \\ Not applicable.}

\section{Authors' contributions}

MW, JEW and JET performed the analysis. MW, JET and SHF developed the stochastic model. MW, JEW, JET, WEO, KEH, MP, RLP and RMA contributed to both the design of the research and the writing of the paper. All authors read and approved the final manuscript.

\section{Funding}

JEW, JET, MW and RMA gratefully thank the Bill \& Melinda Gates Foundation for research grant support via the DeWorm3 (OPP1129535) award to the Natural History Museum in London. JEW, JET, MW and RMA acknowledge joint Centre funding from the UK Medical Research Council and Department for International Development (MR/R015600/1). SHF gratefully acknowledges funding of the NTD Modelling Consortium by the Bill \& Melinda Gates Foundation in partnership with the Task Force for Global Health. WEO, KEH and RLP gratefully acknowledge funding received from the Bill \& Melinda Gates Foundation, the Joint Global Health Trials Scheme of the Medical Research Council, the UK Department for International Development and the Wellcome Trust and the Children's Investment Fund Foundation. The views, opinions, assumptions, or any other information set out in this article are solely those of the authors.

\section{Availability of data and materials}

Data supporting the conclusions of this article are included within the article and its additional files. Data from the simulation runs are available upon request from the corresponding author. All other data used in the analyses are included in the cited publications.

\section{Ethics approval and consent to participate}

Not applicable.

\section{Consent for publication}

Not applicable.

\section{Competing interests}

RMA was a Non-Executive Director of GlaxoSmithKline (GSK) when the TUMIKIA study was conducted. GlaxoSmithKline played no role in study design, data collection and analysis, decision to publish, or preparation of the manuscript. MW, JEW, JET, WEO, KEH, MP, SHF and RLP declare that they have no competing interests.

\section{Author details}

${ }^{1}$ London Centre for Neglected Tropical Disease Research (LCNTDR), Department of Infectious Disease Epidemiology, St. Mary's Campus, Imperial College London, London, UK. ${ }^{2}$ The DeWorm3 Project, The Natural History Museum of London, London, UK. ${ }^{3}$ MRC Centre for Global Infectious Disease Analysis, School of Public Health, Imperial College London, London, UK.

${ }^{4}$ Present Address: Centre for Global Child Health, Hospital for Sick Children, Toronto, Canada. ${ }^{5}$ Faculty of Infectious and Tropical Diseases, London School of Hygiene \& Tropical Medicine, London, UK. ${ }^{6}$ Department of Life Sciences, Natural History Museum, London, UK.

Received: 18 February 2020 Accepted: 25 May 2020

Published online: 08 June 2020

\section{References}

1. Murray CJL, Vos T, Lozano R, Naghavi M, Flaxman AD, Michaud C, et al. Disability-adjusted life years (DALYs) for 291 diseases and injuries in 21 regions, 1990-2010: a systematic analysis for the Global Burden of Disease Study 2010. Lancet. 2012;380:2197-223.

2. Pullan RL, Smith JL, Jasrasaria R, Brooker SJ. Global numbers of infection and disease burden of soil transmitted helminth infections in 2010. Parasites Vectors. 2014;7:37.

3. Hotez PJ, Alvarado M, Basáñez M-G, Bolliger I, Bourne R, Boussinesq M, et al. The Global Burden of Disease Study 2010: interpretation and implications for the neglected tropical diseases. PLoS Negl Trop Dis. 2014;8:e2865.

4. Horton J. Albendazole: a review of anthelmintic efficacy and safety in humans. Parasitology. 2000;121(Suppl.):113-32.

5. Speich B, Ali SM, Ame SM, Bogoch II, Alles R, Huwyler J, et al. Efficacy and safety of albendazole plus ivermectin, albendazole plus mebendazole, albendazole plus oxantel pamoate, and mebendazole alone against Trichuris trichiura and concomitant soil-transmitted helminth infections: a four-arm, randomised controlled trial. Lancet Infect Dis. 2015;15:277-84.

6. Keiser J, Utzinger J. Efficacy of current drugs against soil-transmitted helminth infections: systematic review and meta-analysis. JAMA. 2008;299:1937-48.

7. WHO. Accelerating work to overcome the global impact of neglected tropical diseases: a roadmap for implementation: executive summary. 
Geneva: World Health Organization; 2012. https://apps.who.int/iris/handl e/10665/70809.

8. Mupfasoni D, Mikhailov A, Mbabazi P, King J, Gyorkos TW, Montresor A. Estimation of the number of women of reproductive age in need of preventive chemotherapy for soil-transmitted helminth infections. PLoS Negl Trop Dis. 2018;12:e0006269.

9. London Declaration on Neglected Tropical Diseases. 2012. https://uniti ngtocombatntds.org/london-declaration-neglected-tropical-diseases/. Accessed 22 May 2020.

10. Truscott JE, Hollingsworth T, Brooker SJ, Anderson RM. Can chemotherapy alone eliminate the transmission of soil transmitted helminths? Parasites Vectors. 2014;7:266

11. Anderson RM, Turner HC, Truscott JE, Hollingsworth TD, Brooker SJ. Should the goal for the treatment of soil transmitted helminth (STH) infections be changed from morbidity control in children to communitywide transmission elimination? PLoS Negl Trop Dis. 2015;9:e0003897.

12. Anderson RM, Truscott JE, Pullan RL, Brooker SJ, Hollingsworth TD. How effective is school-based deworming for the community-wide control of soil-transmitted helminths? PLoS Negl Trop Dis. 2013;7:e2027.

13. Anderson R, Truscott J, Hollingsworth TD. The coverage and frequency of mass drug administration required to eliminate persistent transmission of soil-transmitted helminths. Philos Trans R Soc Lond B Biol Sci. 2014;369:20130435.

14. Means AR, Ásbjörnsdóttir K, Mwandawiro C, Rollinson D, Jacobson J, Littlewood T, et al. Sustaining progress towards NTD elimination: an opportunity to leverage lymphatic filariasis elimination programs to interrupt transmission of soil-transmitted helminths. PLoS Negl Trop Dis. 2016;10:e0004737.

15. Jourdan PM, Montresor A, Walson JL. Building on the success of soiltransmitted helminth control-the future of deworming. PLoS NegI Trop Dis. 2017:11:e0005497.

16. Ásbjörnsdóttir KH, Means AR, Werkman M, Walson JL. Prospects for elimination of soil-transmitted helminths. Curr Opin Infect Dis. 2017;30:482-8.

17. Farrell SH, Coffeng LE, Truscott JE, Werkman M, Toor J, de Vlas SJ, et al. Investigating the effectiveness of current and modified World Health Organization guidelines for the control of soil-transmitted helminth infections. Clin Infect Dis. 2018;66(Suppl. 4):253-9.

18. Ásbjörnsdóttir KH, Ajjampur SSR, Anderson RM, Bailey R, Gardiner I, Halliday KE, et al. Assessing the feasibility of interrupting the transmission of soil-transmitted helminths through mass drug administration: the DeWorm3 cluster randomized trial protocol. PLoS Negl Trop Dis. 2018;12:e0006166.

19. Truscott JE, Werkman M, Wright JE, Farrell SH, Sarkar R, Ásbjörnsdóttir K, et al. Identifying optimal threshold statistics for elimination of hookworm using a stochastic simulation model. Parasites Vectors. 2017;10:321.

20. Werkman M, Wright JE, Truscott JE, Easton AV, Oliveira RG, Toor J, et al. Testing for soil-transmitted helminth transmission elimination: analysing the impact of the sensitivity of different diagnostic tools. PLoS Negl Trop Dis. 2018;12:e0006114.

21. Werkman M, Toor J, Vegvari C, Wright JE, Truscott JE, Ásbjörnsdóttir KH, et al. Defining stopping criteria for ending randomized clinical trials that investigate the interruption of transmission of soil-transmitted helminths employing mass drug administration. PLoS Negl Trop Dis. 2018;12:e0006864.

22. Elkins DB, Haswell-Elkins M, Anderson RM. The epidemiology and control of intestinal helminths in the Pulicat Lake region of southern India. I. Study design and pre- and post-treatment observations on Ascaris lumbricoides infection. Trans R Soc Trop Med Hyg. 1986;80:774-92.

23. Hall A, Anwar KS, Tomkins A, Rahman L. The distribution of Ascaris lumbricoides in human hosts: a study of 1765 people in Bangladesh. Trans R Soc Trop Med Hyg. 1999;93:503-10.

24. Seo BS, Cho SY, Chai JY. Frequency distribution of Ascaris lumbricoides in rural Koreans with special reference on the effect of changing endemicity. Korean J Parasitol. 1979;17:105-13.

25. Bradley M, Chandiwana SK, Bundy DAP, Medley GF. The epidemiology and population biology of necator americanus infection in a rural community in Zimbabwe. Trans R Soc Trop Med Hyg. 1992;86:73-6.

26. Guyatt HL, Bundy DAP. Estimating prevalence of community morbidity due to intestinal helminths: prevalence of infection as an indicator of the prevalence of disease. Trans R Soc Trop Med Hyg. 1991;85:778-82.
27. Anderson RM, May RM. Infectious diseases of humans: dynamics and control. Oxford: Oxford University Press; 1991.

28. Anderson R, Farrell S, Turner H, Walson J, Donnelly CA, Truscott J. Assessing the interruption of the transmission of human helminths with mass drug administration alone: optimizing the design of cluster randomized trials. Parasites Vectors. 2017;10:93

29. Dyson L, Stolk WA, Farrell SH, Hollingsworth TD. Measuring and modelling the effects of systematic non-adherence to mass drug administration. Epidemics. 2017;18:56-66.

30. Farrell SH, Truscott JE, Anderson RM. The importance of patient compliance in repeated rounds of mass drug administration (MDA) for the elimination of intestinal helminth transmission. Parasites Vectors. 2017;10:291.

31. Plaisier AP, Stolk WA, van Oortmarssen GJ, Habbema JD. Effectiveness of annual ivermectin treatment for Wuchereria bancrofti infection. Parasitol Today. 2000;16:298-302.

32. Pullan RL, Halliday KE, Oswald WE, Mcharo C, Beaumont E, Kepha S, et al. Effects, equity, and cost of school-based and community-wide treatment strategies for soil-transmitted helminths in Kenya: a cluster-randomised controlled trial. Lancet. 2019;393:2039-50.

33. Brooker SJ, Mwandawiro CS, Halliday KE, Njenga SM, Mcharo C, Gichuki PM, et al. Interrupting transmission of soil-transmitted helminths: a study protocol for cluster randomised trials evaluating alternative treatment strategies and delivery systems in Kenya. BMJ Open. 2015;5:e008950.

34. Truscott JE, Ower AK, Werkman M, Halliday K, Oswald WE, Gichuki PM, et al. Heterogeneity in transmission parameters of hookworm infection within the baseline data from the TUMIKIA study in Kenya. Parasites Vectors. 2019;12:442.

35. Anderson RM, May RM. Population dynamics of human helminth infections: control by chemotherapy. Nature. 1982;297:557-63.

36. Anderson RM, Medley GF. Community control of helminth infections of man by mass and selective chemotherapy. Parasitology. 1985;90:629-60.

37. Truscott JE, Turner HC, Farrell SH, Anderson RM. Soil-transmitted helminths: mathematical models of transmission, the impact of mass drug administration and transmission elimination criteria. Adv Parasitol. 2016:94:133-98.

38. Anderson RM, Gordon DM. Processes influencing the distribution of parasite numbers within host populations with special emphasis on parasite-induced host mortalities. Parasitology. 1982;85:373-98.

39. Bliss Cl, Fisher RA. Fitting the negative binomial distribution to biological data. Biometrics. 1953;9:176.

40. Vercruysse J, Behnke JM, Albonico M, Ame SM, Angebault C, Bethony $J M$, et al. Assessment of the anthelmintic efficacy of albendazole in school children in seven countries where soil-transmitted helminths are endemic. PLoS Negl Trop Dis. 2011;5:e948.

41. Forrester JE, Scott ME. Measurement of Ascaris lumbricoides infection intensity and the dynamics of expulsion following treatment with mebendazole. Parasitology. 1990;100:303-8.

42. Nikolay B, Brooker SJ, Pullan RL. Sensitivity of diagnostic tests for human soil-transmitted helminth infections: a meta-analysis in the absence of a true gold standard. Int J Parasitol. 2014;44:765-74.

43. Benjamin-Chung J, Pilotte N, Ercumen A, Grant JR, Maasch JRMA, Gonzalez AM, et al. Comparison of multi-parallel qPCR and Kato-Katz for detection of soil-transmitted helminth infection among children in rural Bangladesh. PLoS NegI Trop Dis. 2020;14:e0008087.

44. Easton AV, Oliveira RG, O'Connell EM, Kepha S, Mwandawiro CS, Njenga SM, et al. Multi-parallel qPCR provides increased sensitivity and diagnostic breadth for gastrointestinal parasites of humans: field-based inferences on the impact of mass deworming. Parasites Vectors. 2016;9:38.

45. Wright JE, Werkman M, Dunn JC, Anderson RM. Current epidemiological evidence for predisposition to high or low intensity human helminth infection: a systematic review. Parasites Vectors. 2018;11:65.

46. Vlaminck IdJ, Cools P, Albonico M, Ame S, Ayana M, Bethony J, et al. Comprehensive evaluation of stool-based diagnostic methods and benzimidazole resistance markers to assess drug efficacy and detect the emergence of anthelmintic resistance: a starworms study protocol. PLoS Negl Trop Dis. 2018;12:e0006912.

47. Easton AV, Dahlstrom E, Porcella SF, Oliveira RG, Wang J, Gao S, et al. Whole genome sequencing of Ascaris lumbricoides worms reveals changes in space and time. Am J Trop Med Hyg. 2017;5:598-9.

48. Vegvari C, Truscott JE, Kura K, Anderson RM. Human population movement can impede the elimination of soil-transmitted helminth 
transmission in regions with heterogeneity in mass drug administration coverage and transmission potential between villages: a metapopulation analysis. Parasites Vectors. 2019;1 2:438.

49. Turner HC, Truscott JE, Bettis AA, Shuford KV, Dunn JC, Hollingsworth TD, et al. An economic evaluation of expanding hookworm control strategies to target the whole community. Parasites Vectors. 2015;8:570.
50. Bethony J, Brooker SJ, Albonico M, Geiger S, Loukas A, Diemert D, et al. Soil-transmitted helminth infections: ascariasis, trichuriasis, and hookworm. Lancet. 2006;367:1521-32.

51. Anderson RM, May RM. Helminth infections of humans: mathematical models, population dynamics, and control. Adv Parasitol. 1985;24:1-101.

52. Coffeng LE, Truscott JE, Farrell SH, Turner HC, Sarkar R, Kang G, et al. Comparison and validation of two mathematical models for the impact of mass drug administration on Ascaris lumbricoides and hookworm infection. Epidemics. 2017;18:38-47.

\section{Publisher's Note}

Springer Nature remains neutral with regard to jurisdictional claims in published maps and institutional affiliations.
Ready to submit your research? Choose BMC and benefit from:

- fast, convenient online submission

- thorough peer review by experienced researchers in your field

- rapid publication on acceptance

- support for research data, including large and complex data types

- gold Open Access which fosters wider collaboration and increased citations

- maximum visibility for your research: over 100M website views per year

At BMC, research is always in progress.

Learn more biomedcentral.com/submissions 